\title{
A comparative analysis of traditional dental screening versus teledentistry screening
}

SADJ February 2019, Vol. 74 No. 1 p19 - p23

S Bissessur ${ }^{1}$, S Naidoo ${ }^{2}$

\section{ABSTRACT}

This comparative analysis determined the reliability of using intraoral cameras and communication technologies (ICTs) to screen primary school children for dental caries.

\section{Methods}

A concordance study assessed the diagnostic agreement between traditional and teledentistry screening of dental caries in 233 school children aged 6-8 years old from randomly selected rural primary schools. Clinical screening, scored for DMFT, was carried out by two trained and calibrated examiners. Two trained and calibrated teledentistry assistants (TAs) captured intraoral images, and stored the data in corresponding eFiles. After a two week wash-out period, the intraoral images were evaluated and scored for DMFT. Concordance and inter- and intra-examiner reliability were determined using the Kappa Statistic.

\section{Results}

The intra-rater agreement and reliability across methods was $98.30 \%$ and $95.09 \%$ for examiners one and two respectively, assessed as indicating $92.79 \%$ between random agreement and perfect agreement $(p<0.001)$. The high concordance level indicated that there was no statistical difference between the clinical screening and the screening teledentistry method.

\section{Conclusion}

This study highlights the reliability of utilising teledentistry as a dental screening and diagnostic tool which can be valuable in the delivery of oral health care in South Africa especially with view to the implementation of the $\mathrm{NHI}$.

\section{Keywords}

South Africa, children, dental caries, dental screening, ICT, telemedicine, teledentistry, DMFT, oral health care, eHealth

\section{Author affiliations:}

1. Sabeshni Bissessur: $B O H$ (UDW), BDTh (UDW), MSc (UWC), PhD (UWC), Department of Community Oral Health, University of the Western Cape, Tygerberg.

2. Sudeshni Naidoo: BDS(Lon), LDS.RCS (Eng), MDPH (Lon), DDPH. RCS (Eng), MChD (Comm Dent), PhD (US), Dipl. Int Research Ethics (UCT), Senior Professor and Principal Specialist, Department of Community Oral Health, University of the Western Cape, Tygerberg. Corresponding author: Sabeshni Bissessur

286 Spencer Road, Clare Estate, Durban, 4091. Tel: +27 (0)31 2692891 Email: sabeshb@vodamail.co.za

\section{ACRONYMS \\ ICT: Information and Communication Technology \\ NHI: National Health Insurance \\ TA: $\quad$ Teledentistry Assistants}

\section{BACKGROUND}

Teledentistry is the use of information and communication technology (ICT) to provide oral health care services and enhance oral health care delivery to rural communities that may not have access to health services.

Public health services in South Africa are in the process of being overhauled to address the inadequacies and the use of ICT's has been identified as a possible tool in improving the delivery of health care. Telemedicine has been recognised as having the potential to improve access to health care, but teledentistry does not currently feature in the dental public health sector.

Teledentistry and mobile health delivery have the potential to eliminate or minimise the disparities in oral health care that exist in South Africa. The technique can be initiated in an incremental approach by 'piggy-backing' on existing telemedicine sites, thereby reducing the ICT costs for the public health sector.

Stakeholders and government officials need to embrace technology to address some of the challenges that exist in the South African public health sector. This study provides evidence-based information to assist in the introduction of teledentistry in South Africa as an innovative dental screening and management tool.

The teledentistry screening method used in the present study was the store-and-forward method where the images are web-based stored and later assessed at a remote site. The teledentistry real-time consulting method can be utilized for patients visiting the dental clinics and who require specialist consultations.

Real-time teledentistry consultations usually include a videoconference in which dental professionals and their patients, at different locations, may see, hear, and communicate with one another. ${ }^{1}$

With real-time consultations, also known as synchronous teleconsultations, information is transferred immediately and there is a two-way interaction between the patient and the consulting specialist, which allows for immediate 
feedback from the specialist consultant. Hence, through the use of telecommunications and computer technologies, it is now possible to provide interactive access to specialist opinions in communication which is not limited by the constraints of either space or time. ${ }^{2}$ Specialist care may thus be accessible in critical access areas, a contribution toward bridging the gap between demand and supply.

The most recent SA National Oral Health Survey showed that at least $80 \%$ of dental caries in children is untreated, ${ }^{3}$ posing a significant public health problem.

Teledentistry has been recommended as a school screening tool to reduce the double burden of burgeoning dental caries in children and shortages of human resource in the public sector. ${ }^{4}$ The technique has the potential to improve access and delivery of oral health care to children in underserved and the rural areas.

The traditional method of dental screening usually involves a registered oral health worker who carries out a visual inspection of the child's mouth and teeth by using an intra-oral mirror, a portable chair and a light source.

A registered dental assistant is often required to function as the recorder of the DMFT. ${ }^{5}$ The DMFT index records decayed (D), missing (M) and filled (F) teeth. The recording and calculation of the DMFT score is a globally used and accepted measurement of the prevalence of caries. ${ }^{6}$

The DMFT is usually recorded on paper at the face-to-face dental screening session, and there is a dental charting form or data capture sheet for each child. In addition, infection control must be adhered to at all times.

On the other hand, an intraoral camera is used in teledentistry to screen for dental caries and information and communication technologies are utilised in management of the data. ${ }^{4}$ This paper describes a study that compared traditional dental screening with teledentistry screening for dental caries in children.

\section{METHODS}

The Richmond Municipality is a designated National Health Insurance pilot site, in the uMgungundlovu District of Kwa-Zulu-Natal, South Africa. Richmond is located on the southern part of the uMgungundlovu District Municipality and is approximately 38 kilometers south of Pietermaritzburg (the capital of the KwaZuluNatal Province). ${ }^{7}$ The study was carried out in Magoda, a rural area in Richmond. Three primary schools were randomly selected to participate in the study.

Prior to the initiation of the study, gateway permission to work in the primary schools was obtained from the Department of Education (DoE) and ethical clearance was approved by the Senate Research Ethics Committee at the University of the Western Cape (Ref No. 12/7/20).

In addition, information sheets were used to explain the procedures, possible risks and benefits to the parents/ guardians of the participating children, and written informed consent was obtained for each participant.
For the first phase of the data collection two registered dental clinicians were designated as examiners One and Two. Both clinicians were trained, standardised and calibrated to carry out the face-to-face intra-oral examinations (traditional dental screening) which were conducted on 233 children aged between 6 and 8 years old at the selected three rural primary schools.

Headlamps, disposable tongue depressors, gauze, gloves, masks and disposable plastic picks were used. The latter instruments were used instead of standard periodontal probes to determine pit and fissure caries and to remove debris. In this way, cold sterilization was not required, which in turn saved time and ensured that infection control was maintained at all times. Each examiner independently carried out the examinations of the selected children and scored them for DMFT. A modified WHO Oral Health Assessment Form (1997) was used as the hard copy data capture sheet.

Following the face-to-face examinations, two trained and calibrated teledentistry assistants used an intraoral camera (Kodak 1500 - Carestream) and a lap top computer to capture digital images of the children's teeth. The TAs used gauze to remove any debris from the dentition. A disposable wooden tongue depressor was used to retract the cheeks, lips and the tongue to facilitate clear vision of the teeth. The intraoral camera was focused on the teeth, and the image was simultaneously viewed on a laptop.

Once a clear image was obtained, the image was captured electronically on the laptop. Images of poor quality were deleted and retaken if necessary. The images were web-based stored for each child. Four intraoral images of the upper and lower anterior teeth and the four posterior quadrants were captured (Figure 1). The imaging process was non-invasive, pain-free and there was little or no discomfort to the child. The imaging process took about 10 to 15 minutes per child. Security of data was maintained at all times by the use of protective passwords. Confidentiality and anonymity was maintained as each child was coded and no names were used.

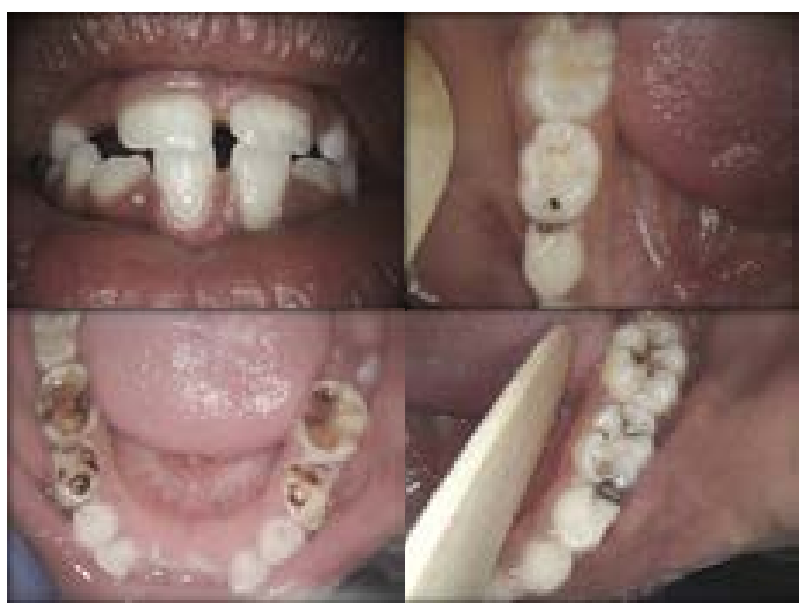

Figure 1. Examples of the images captured by the TAs.

Following phase 1 of the data collection, the examiners had a 'wash out' period of two weeks. Thereafter evaluation of the intraoral images of the teeth was undertaken and scored for DMFT. All intra-oral images were randomized and blinded to keep bias to a minimum. 
The data cleaned and the DMFT scores were verified. The DMFT scores were compared not only across methods (i.e. traditional dental screening (Gold Standard) versus teledentistry screening of dental caries), but also across examiners.

\section{RESULTS}

A total of 233 children were examined by two examiners using two different methods of dental screening, namely the traditional dental screening method and the teledentistry screening method. The resulting data were used to determine concordance and reliability between the two methods.

The study sample consisted of 131 males and 102 females. Overall, the ratio of males to female was 3:2 (56.2\%:43.8\%). The mean age was 6.73 years. There were significantly more male participants aged 6 and 7 years old, while females dominated the 8 year-old category. A high percentage of the children had sound teeth (69\%). The traditional dental screening method found $20 \%$ to have decayed teeth whilst the teledentistry screening method recorded $21 \%$. No children had filled teeth.

The cross-tabulation shown in Table 1 indicates the frequencies of DMFT assessments made by examiner 1 (E1) comparing the traditional dental screening method and teledentistry screening method (Image Assessor-IA1). For example, E1 rated the 1's simultaneously (3863 times) in both dental screening methods.

On 23 occasions, E1 scored a rating of 2 while IA1 scored a rating of 1 . There was an almost perfect agreement of the DMFT assessments obtained from both dental screening methods with a kappa value of 0.9630 (Table 1a).

\begin{tabular}{|c|c|c|c|c|c|c|}
\hline \multirow[b]{2}{*}{ E1 } & \multicolumn{5}{|c|}{ |A1 } & \multirow{2}{*}{ Total } \\
\hline & 1 & 2 & 5 & 6 & 7 & \\
\hline 1 & 3863 & 50 & 1 & 5 & 2 & 3921 \\
\hline 2 & 23 & 1177 & 2 & 2 & 0 & 1204 \\
\hline 5 & 2 & 0 & 219 & 0 & 1 & 222 \\
\hline 6 & 0 & 0 & 0 & 0 & 0 & 0 \\
\hline 7 & 3 & 0 & 0 & 4 & 229 & 236 \\
\hline Total & 3891 & 1227 & 222 & 11 & 232 & 5583 \\
\hline
\end{tabular}

\begin{tabular}{|c|c|c|c|c|c|}
\hline Agreement & $\begin{array}{l}\text { Expected } \\
\text { Agreement }\end{array}$ & Kappa & Std. Err. & Z & Prob $>Z$ \\
\hline $98.30 \%$ & $54.02 \%$ & 0.9630 & 0.0103 & 93.12 & 0.0000 \\
\hline
\end{tabular}

The cross-tabulation in Table 2 indicates the frequency of DMFT assessments made by E2 comparing the traditional dental screening method and teledentistry screening method (Image Assessor-IA2). For example, E2 rated the 1's simultaneously (3779 times) in both dental screening methods.

On 90 occasions, E2 scored a rating of 2 whilst IA2 scored a rating of 1 . There was an almost perfect agreement of the DMFT assessments obtained from both dental screening methods with a kappa statistic of 0.8926 (Table 2a).

\begin{tabular}{|c|c|c|c|c|c|c|c|}
\hline \multirow[b]{2}{*}{ E1 } & \multicolumn{6}{|c|}{ IA2 } & \multirow{2}{*}{ Total } \\
\hline & 1 & 2 & 4 & 5 & 6 & 7 & \\
\hline 1 & 3779 & 142 & 1 & 7 & 4 & 4 & 3937 \\
\hline 2 & 90 & 1071 & 0 & 4 & 2 & 0 & 1167 \\
\hline 4 & 0 & 0 & 0 & 0 & 0 & 0 & 0 \\
\hline 5 & 7 & 0 & 0 & 212 & 0 & 0 & 219 \\
\hline 6 & 0 & 0 & 0 & 0 & 0 & 0 & 0 \\
\hline 7 & 7 & 1 & 0 & 0 & 4 & 229 & 241 \\
\hline Total & 3883 & 1214 & 1 & 223 & 10 & 233 & 5564 \\
\hline
\end{tabular}

\begin{tabular}{|c|c|c|c|c|c|}
\hline Agreement & $\begin{array}{l}\text { Expected } \\
\text { Agreement }\end{array}$ & Kappa & Std. Err. & Z & Prob $>$ Z \\
\hline $95.09 \%$ & $54.30 \%$ & 0.8926 & 0.0103 & 86.46 & 0.0000 \\
\hline
\end{tabular}

The intra-rater agreement and reliability for both examiners across the dental screening methods gave a kappa value of 0.9630 (98.30\%) for E1 and 0.8926 (95.09\%) for E2. These kappa values show an almost perfect intra-rater agreement of the DMFT assessments obtained across dental screening methods for both examiners.

The overall inter-rater agreement and reliability for the traditional screening method revealed a kappa value of 0.9279 (96.73\%). There was a similar high level inter-rater agreement and reliability for the teledentistry screening method with a kappa value of 0.9480 (97.59\%). Both the intra-rater and inter-rater values show high agreement with almost perfect agreement $(p<0.001)$.

\section{DISCUSSION}

In this concordance study, traditional face-to-face DMFT scores and teledentistry DMFT scores were compared for diagnostic agreement and reliability. There was a high frequency of agreement between traditional dental screening and teledentistry screening. Concordance between teledentistry and traditional dental screening methods ranged from $95.09 \%$ to $98.30 \%$ for both examiners implying that the use of teledentistry for facilitating oral health screening can be effective.

The inter-rater analysis comparing the DMFT assessments made by both examiners (E1and E2) were found to be in almost perfect agreement for both the traditional and teledentistry screening methods. The frequency of decayed teeth scored using the traditional dental screening method was $20 \%$ and for the teledentistry screening $21 \%$ - again suggesting high agreement across methods.

The fact that both examiners had similar training, academic background and years of clinical experience, could have impacted positively on the results obtained and also suggests that they were competent to determine DMFT using both dental screening methods. However, this may not always be the case.

Decision support systems or diagnostic aids have been widely implemented to assist dental health professionals in making diagnoses using the ICT platform. If there are standard assessment tool/diagnostic protocols, this 
will permit all dental professionals to utilise the teledentistry tool regardless of the level of experience. Other fields of telemedicine have utilised decision support systems successfully. ${ }^{8}$

The high concordance levels indicated that there was no statistical difference between the traditional dental screening method and the teledentistry screening method (intra-rater reliability), thus suggesting that the teledentistry screening method is a reliable alternative to the traditional dental screening method. Furthermore, this implies that intraoral images are a reliable tool to diagnose decayed, missing and filled teeth in children.

There was a high percentage of children with sound teeth and less than a quarter of children presented with decayed teeth. No children had filled teeth and many reported that they had their teeth examined for the first time during the present study.

The most recent South African National Oral Health Survey (NOHS) revealed that 80 per cent of carious lesions in children go untreated, ${ }^{3,9}$ which highlights the challenges of meeting the demanding dental needs for South Africa's children, especially those living in remote and rural areas. This burden is compounded by the shortage of dental professionals that are required to provide a service to large populations.

Disagreement between the examiners was minimal across the different methods and this could be attributed to a few factors. Firstly, no radiographs were taken making pit and fissure caries difficult to determine in both methods. Secondly, the intraoral teledentistry images can be magnified with good lighting thus improving visualisation and enhancing diagnosis of sound or decayed teeth (with an exception for pit and fissure caries). It is also important to note that the intraoral images are purposefully captured to provide the assessors with the best quality to enable accurate diagnosis. Thirdly, interproximal caries is not easy to detect with either the traditional or teledentistry dental screening method unless it is obviously visible, such as a break in the surface enamel or the presence of large areas of shadowing.

The significance of the findings of the present study is that teledentistry can be used as a valuable alternate to the traditional dental screening face-to-face method especially when screening large populations of children in rural areas, particularly in Sub-Saharan Africa where poverty and the delivery of oral healthcare is a great challenge. South Africa is in an on-going process in the endeavour to address the disparities within the health system.

One of the key aims of the NHI is to re-engineer the way in which school-based primary health care services are provided, and teledentistry has a significant role to play. In this regard, it may be a strategic opportunity to introduce the technique into the school-based primary health care policy, so as to improve oral health promotion and service delivery.

Panat et al found teledentistry to be a good tool for screening dental caries in schoolchildren, enabling their differentiation into categories of high risk or low risk patients. ${ }^{10}$ This risk triage can assist dental professionals in tailoring curative and preventive packages to assist communities in combating dental caries in schoolchildren.

Young children need to be aware of the importance of good oral health so that they can prevent dental caries, early tooth loss and other oral diseases. Risk assessment is now viewed as the critically important step in the clinical decision-making process of managing dental caries. ${ }^{11}$ For teledentistry, caries risk assessment can effectively manage and triage patients for treatment planning, prevention, and establishing follow-up and recall times.

The combination of teledentistry with the caries risk assessment strategy can be significant in providing a synergy between preventive and curative oral health care for children in critical access communities. ${ }^{11}$ Therefore, teledentistry should be strategically engineered into the school oral health screening programmes.

The categorization of the caries risk factors will then allow for focused recommendations to minimise the risk of children developing new carious lesions and/or the progression of existing lesions in the future, ${ }^{12}$ thereby promoting oral health care and preventing further disease. This further highlights the potential value of the teledentistry screening method as it may be envisaged as a more cost-effective method to screen large populations of children.

The proposed introduction of the National Health Insurance (NHI) aims to ensure that everyone has access to appropriate, efficient and quality health services regardless of their socio-economic status. ${ }^{13}$ Appropriateness is one of the guiding principles of the $\mathrm{NHI}$ and refers to adopting new and innovative health service delivery models that focus on local needs. ${ }^{13}$ It may be a viable option for the Department of Health to consider teledentistry for implementation into the $\mathrm{NHI}$ and the next National Oral Health Survey (NOHS). In addition, one of the objectives of the $\mathrm{NHI}$ is to strengthen the under-resourced and strained public sector so as to improve health systems performance. ${ }^{13}$ Teledentistry is an intervention that can enhance opportunities for the new primary healthcare model and has the capacity to take service delivery and accessibility to new and improved levels.

The teledentistry screening method will eliminate the need for instrumentation (like mouth mirrors and probes) and infection control as cold sterilization of the instruments will not be required. Furthermore, National Oral Health Surveys often require the deployment of a large number of calibrated dental professionals to carry out visual faceto-face examinations. Teledentistry screening can utilise trained and calibrated non-dental personnel (community health workers or nurses for example that are already based in the communities) to capture the intraoral images of the school children and the web-based stored images can then be assessed by the dental professionals at a distant site in their own time (community health dentists can be utilised for this role). Therefore, teledentistry screening can potentially greatly reduce the human resources required and save travel time so that dental professionals make better use of their clinical training and expertise in the dental clinics. 


\section{CONCLUSION}

The present study has shown the reliability of teledentistry as a dental screening tool. Once initiated, teledentistry has the potential to grow and develop within its own versatility and various adaptations. For example, it can be used for a variety of online dental information and education purposes, for electronic consumer medical and health information, dentist-laboratory communication, patient consultations, specialist referrals and for remote patient monitoring or home-health care. All of which have great potential to enhance dental education and importantly to enhance the delivery of oral health care in South Africa.

\section{References}

1. Bhambal A, Saxena S and Balsaraf SV. Teledentistry: potentials unexplored. J Int Oral Health. 2010; 2:1-6.

2. Jain A, Bhaskar DA, Gupta D, Agali C, Gupta V, Karim B. Teledentistry: upcoming trend in Dentistry. J Adv Med Dent Scie. 2013; 1(2):112-5.

3. Department of Health. Report: National Children's Oral Health Survey. South Africa; 2003.

4. Kopycka-kedzierawski DT, Billings RJ and McConnochie KM. Dental screening of preschool children using teledentistry: a feasibility study. Paediatr Dent. 2007; 29(3):209-13.

5. Patterson S, Botchway C. Dental screenings using Telehealth Technology: a pilot study. J Can Dent Assoc. 1998; 64:806-10.

6. Bratthall D. Introducing the Significant Caries Index together with a proposal for a new global oral health goal for 12 year olds. Inter Dental Journal. 2000; 50(6):378-84.

7. Ricmond Municipality Draft Integrated Development Plan-2012/2013 to 2016/2017. Richmond Municipality Development and Planning Unit 2012; 1-156.

8. Moore J. A cataract decision support system: its requirement is increasing. Stud Health Technol Inform. 2005; 117:147-53.

9. Van Wyk PJ, Van Wyk C. Oral health in South Africa. International Dental Journal 2004; 54:373-7.

10. Panat SR, Chakarvaty A, Aggarwal A. Teledentistry: A new revolution. Journal of Dental Sciences \& Oral Rehabilitation 2012.

11. Van Hilsen, Jones RS. Comparing potential early caries assessment methods for Teledentistry. BMC Oral Health 2013; 13(16)

12. Pitts N, Melo P, Martignon S, Ekstrand K, Ismail A. Caries risk assessment. Diagnosis and synthesis in the context of a European core curriculum in Cariology. Eur J Den Edu. 2011; 15(1): 23-31.

13. Department of Health. National Health Insurance in South Africa: Policy Paper. 2011.

\section{ERRATUM}

The November issue of the Journal carried an article which was not correctly categorised. The paper Cervical restorations useful when assessing gingival and periodontal health was published on page 633 under the category Industry News.

WG Evans: Managing editor, Email: bill.evans@wits.ac.za
The correct category is Clinical Communication. Sincere apologies to the author.

Reference: 2018;73(10):633. 